ORIGINAL ARTICLE

\title{
Making psychological theory useful for implementing evidence based practice: a consensus approach
}

\author{
S Michie, M Johnston, C Abraham, R Lawton, D Parker, A Walker, on behalf of the "Psychological \\ Theory" Group
}

Qual Saf Health Care 2005;14:26-33. doi: 10.1136/qshc.2004.011155

See end of article for authors' affiliations

Correspondence to:

Correspondence to:
Dr S Michie, Centre for Outcomes Research and Effectiveness, Department of Psychology, University College London, London WCIE 7HB, UK; s.michie@ucl.ac.uk

Accepted for publication 12 November 2004
Background: Evidence-based guidelines are often not implemented effectively with the result that best health outcomes are not achieved. This may be due to a lack of theoretical understanding of the processes involved in changing the behaviour of healthcare professionals. This paper reports the development of a consensus on a theoretical framework that could be used in implementation research. The objectives were to identify an agreed set of key theoretical constructs for use in (1) studying the implementation of evidence based practice and (2) developing strategies for effective implementation, and to communicate these constructs to an interdisciplinary audience.

Methods: Six phases of work were conducted to develop a consensus: (1) identifying theoretical constructs; (2) simplifying into construct domains; (3) evaluating the importance of the construct domains; (4) interdisciplinary evaluation; (5) validating the domain list; and (6) piloting interview questions. The contributors were a "psychological theory" group $(n=18)$, a "health services research" group $(n=13)$, and a "health psychology" group $(\mathrm{n}=30)$.

Results: Twelve domains were identified to explain behaviour change: (1) knowledge, (2) skills, (3) social/ professional role and identity, (4) beliefs about capabilities, (5) beliefs about consequences, (6) motivation and goals, (7) memory, attention and decision processes, (8) environmental context and resources, (9) social influences, (10) emotion regulation, (11) behavioural regulation, and (12) nature of the behaviour. Conclusions: A set of behaviour change domains agreed by a consensus of experts is available for use in implementation research. Applications of this domain list will enhance understanding of the behaviour change processes inherent in implementation of evidence-based practice and will also test the validity of these proposed domains.
$\mathrm{R}$ ecommendations for evidence based practice (EBP) are frequently published following systematic reviews of evidence (for example, from the Cochrane collaboration and the UK National Institute of Clinical Excellence). However, often evidence-based guidelines are not implemented effectively with the result that best health outcomes are not achieved. ${ }^{1}$ A practical example of the problem of implementing EBP in the area of hand hygiene is described in box 1 .

It has been agreed that there is no "magic bullet" to changing professional practice, ${ }^{2}$ and that the effectiveness of strategies is sensitive to context. ${ }^{3}$ The mixed results and the limited practical value of much of this research may be due to a limited theoretical basis for the development of interventions. The atheoretical nature of many of the interventions is unsurprising, given the large and sometimes overlapping array of psychological theories and component parts of theories (that is, theoretical "constructs") that could be used.

More than 230 methodologically adequate studies of the effectiveness of guideline dissemination and implementation strategies have been identified. ${ }^{4}$ The behaviour change methods used have been mainly intuitive or educational. These have included printed materials, audit and feedback, reminders, and educational outreach. ${ }^{5}$ While small to moderate effects have been observed both within and between methods, there is no clear pattern of results favouring any one particular method. ${ }^{2}$ There is also no basis for understanding which procedures are effective in which contexts because it is unclear how successful interventions have their effect-that is, what behaviour change processes are responsible for observed change. Thus, despite the large number of studies, there is no basis for selecting an intervention with confidence.

Previous attempts to identify theoretical models explaining behaviour change have generated a plethora of contending theories. ${ }^{6}$ Various authors have suggested a range of theories that might be used, ${ }^{78}$ and Ashford's review identifies some 20 possible theories, each with many theoretical constructs, developed within the social and behavioural sciences. There are two problems with drawing from such a large pool of theories and theoretical constructs: firstly, critical theories may be missed and, secondly, such a large number of theories cannot be fully applied and there is no good basis for selecting among them. Ideally, researchers should have ready access to a definitive set of theoretical explanations of behaviour change and a means of identifying which are relevant to particular contexts. In this paper we describe an attempt to reach expert consensus on which theoretical constructs should be included and applied.

Psychological theories are numerous and many have shared or overlapping constructs. Bandura ${ }^{9}$ has commented on the potential for idiosyncratic selection of constructs. Constructs may be renamed to suggest different constructs or a single construct may be differentiated into multiple constructs. For example, many theories describe the individual's perception of control over their behaviour or environment. In a review of the perceived control construct Skinner ${ }^{10}$ lists over 100 conceptualisations. This range of theoretical elaboration makes it difficult to know how to select and apply psychological theories.

Clarification and simplification are necessary to maximise the accessibility and usefulness of psychological theory. The 
Box 1 Hand hygiene: a practical example of the problem of implementing evidence based practice

In 2002 a report by the Chief Medical Officer stressed that hand hygiene was the most important factor in preventing infections associated with health care. Aftempts have been made to increase this simple activity, many based on increasing the numbers of sinks and making an alcohol based rub more widely available. A recent study of hand hygien ${ }^{25}$ in an emergency department found that hand hygiene was poor, particularly between consultations and even when the procedures were non-urgent. The approach adopted in this paper acknowledges the organisational constraints (resources, facilities, time) that mitigate against changes in evidence-based practices such as hand washing. At the same time, the approach acknowledges that psychological factors also have a role in precipitating or stifling behaviour change. For example, at the motivational level, it may be that local group norms clearly prioritise throughput rather than hygiene, and repeated hand washing is seen as obsessive. Alternatively, there may be an attitude amongst staff that hand hygiene has little to do with the rates of infection (beliefs about the consequences). Finally, the problem in making the change may be in action initiationfor example, healthcare staff are aware of the need for hand washing but forget to carry it out. In this case, local cues to action-for example, patient reminders to staff, may be necessary to increase rates. The solution to implementing the evidence-based practice very clearly depends on the problem. The development of the framework described here attempts to do just this - it helps identify and understand the problem. ${ }^{26}$

success of future implementation research is likely to be enhanced by applying psychological explanations of behaviour change rather than models that simply predict behaviour. Consequently, a useful first step would be to identify those theories and component parts of theories-that is, theoretical "constructs" that relate to behaviour change. These theories are likely to identify opportunities and methods for intervention.

A previous theory simplification exercise by leading psychological theorists Fishbein, ${ }^{11}$ Bandura, ${ }^{12}$ Triandis, ${ }^{13}$ Kanfer, ${ }^{14}$ and Becker $^{15}$ led to a consensus on constructs relevant to HIV research on behaviour. However, it is not clear how this consensus was reached or how relevant these constructs might be for research on the implementation of EBP. Furthermore, the framework developed by Fishbein et $a l^{16}$ was published for a psychology readership. Since research on the implementation of EBP is frequently conducted by non-psychologists, it would be valuable to make such a framework accessible to non-psychologists.

This paper presents the method and results of a project which aimed to identify an agreed set of key theoretical constructs for use in (1) studying the implementation of EBP and (2) developing strategies for effective implementation, and to communicate these constructs to an interdisciplinary audience.

\section{METHODS}

\section{Contributors}

Three groups of experts were identified and were invited to contribute to the project.

As well as the main working group of health psychology theorists, two further groups played distinctive roles in refining and validating the findings: a multidisciplinary group was asked to give feedback on the utility of the approach to health services research, and a psychological group to carry out a backward validation exercise.

\section{Health psychology theorists}

Health psychology theorists (theory group) with an interest in theoretical approaches to implementation research and behaviour change had the task of identifying theoretical constructs and reaching a consensus about how related theoretical constructs are best grouped into categories or "domains". This psychological "theory group" comprised 18 UK health psychologists with a range of research interests in organisational, clinical, social and public health psychology. One third of the group had experience of working as clinical or organisational consultants within the NHS.

\section{Health services researchers}

Health services researchers involved in implementation research were included to give critical feedback on the proposed list of construct domains. The "health service research (HSR) group" comprised 16 implementation researchers from an international research collaboration funded by the UK Medical Research Council. It comprised three general practitioners, one public health specialist, two dentists, one pharmacist, three statisticians, one health service researcher, three sociologists, and two psychologists. ${ }^{*}$ Three were from the Ottawa Health Research Institute in Canada and one was from the Centre for Quality of Care Research, Nijmegen, the Netherlands.

\section{Health psychologists}

Health psychologists without a specific expertise in theory, implementation research or behaviour change were included as a validation exercise. The "health psychology (HP) group" comprised 30 delegates at a national health psychology conference.

\section{Procedure}

The work was carried out in a series of five meetings, with intervening homework tasks, between May 2003 and July 2004. The procedural steps are summarised in fig 1 . They were planned and facilitated by the first two authors and records were kept of all meetings and tasks. There were six stages to the work:

- identifying theories and theoretical constructs (theory group);

- simplifying into theoretical domains (theory group);

- evaluating the importance of the theoretical domains (theory group);

- interdisciplinary evaluation (theory group with HSR group);

- validating the domain list (HP group); and

- pilot interview questions (theory group alone and then with HSR group).

\section{Identifying theories and theoretical constructs (theory group);}

In an initial brainstorming session, participants identified as many psychological theories and theoretical constructs as possible that were relevant to EBP implementation. Theoretical constructs were defined as component parts of

*Jill Burrough, Marion Campbell, Jan Clarkson, Martin Eccles, Rhona Flin, Robbie Foy, lan Graham, Jeremy Grimshaw, Graeme MacLennan, Nigel Pitts, Liz Shirran, Nick Steen, Jacqueline Tetroe, Mags Watson, Michel Wensing, Paula Whitty. 


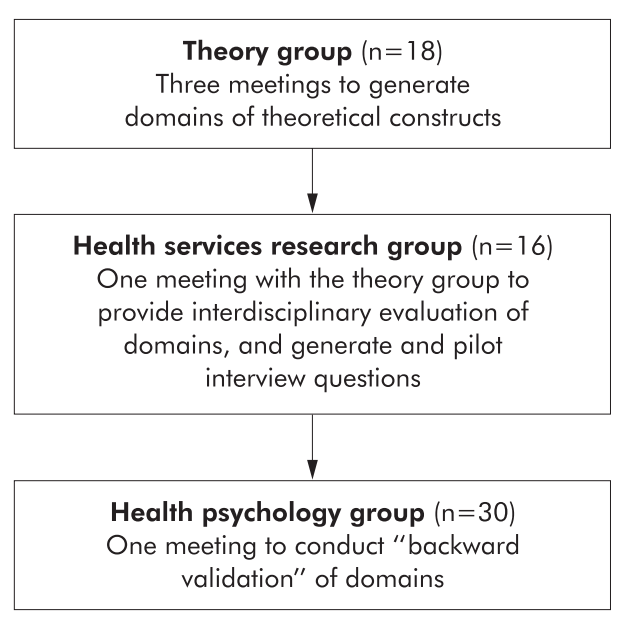

Figure 1 Flow chart to illustrate the procedural steps.

theories. For example, the theory of planned behaviour ${ }^{17}$ incorporates the constructs of "attitude" and "norms".

Participants were asked to identify theories and constructs in each of the following three groups and to attend to explanations of both behaviour and behaviour change:

- motivational: theories to explain behaviour change in people who have not yet established an intention to engage in a particular behaviour;

- action: theories to explain the behaviour of those who are motivated to change; and

- organisational: theories to explain change at a higher order social and systems level. ${ }^{18}$

When a final list had been drawn up, participants were given time to consider whether relevant theories and constructs were omitted.

Simplifying into theoretical domains (theory group) Following the first meeting, participants were each asked to prioritise three of the listed theories and constructs as particularly relevant to (a) understanding and changing behaviour in interdisciplinary research and (b) intervening to change the behaviour of healthcare professionals. The aim of this procedure was to limit the overall number of constructs while allowing for input from a range of perspectives.

In a second meeting two subgroups of the theory group independently grouped these prioritised theories and constructs into core domains. A domain was defined as encompassing a set of similar theoretical constructs. The two groups were asked to limit the number of domains generated to between 5 and 9 as research has shown that short term memory deteriorates markedly when asked to recall more than 7-9 items, and the aim was to create an accessible and memorable summary of behaviour change theory. The two domain lists were compared and a composite summary list agreed through discussion.

Finally, participants of the theory group were asked to allocate each of the theories and constructs to the agreed list of domains. When a construct was assigned to a domain by at least $50 \%$ of group members ${ }^{*}$ it was retained as a construct relevant to the domain.

*The $50 \%$ criterion was arbitrary but a more demanding definition of consensus than a simple majority. If there was little consensus about allocation of a construct to a particular domain, then the greatest number of group members supporting that allocation could be as low as two since the construct could have been assigned to different domains by all other group members.
Evaluating the importance of the theoretical domains (theory group)

Following the second meeting, participants individually evaluated the list of theoretical domains for coherence, for overlapping constructs, and for relevant constructs that had been omitted from the list generated in the first stage. This fed into discussions in two independent subgroups of the theory group and then a plenary discussion, which generated an agreed list of domains encompassing the set of theories and constructs.

Interdisciplinary evaluation (theory group with HSR group)

The theoretical domains were evaluated in relation to their utility to health service researchers, particularly in relation to evaluating behaviour change interventions. Domains were ranked in importance by assessing which were essential, and comparing and matching the domains with a list of factors influencing behaviour that had been generated by a group of leading psychologists in the USA. ${ }^{16}$

\section{Validating the domain list (HP group)}

Having simplified the list of constructs into domains, a "backward validation" exercise was undertaken whereby participants were provided with the list of domains and asked to identify theories and constructs reflecting the content of each domain. Each of the 30 HPs did this individually. In order to identify the key constructs within each of the domains, four groups were then each given three of the domains and asked to agree the three most important constructs in each domain.

\section{Pilot interview questions (theory group alone and} then with HSR group)

Both the theory group and the HSR group were asked to generate interview questions that would (a) assess the nature of behaviour change required to achieve a recommended change in healthcare practice and (b) identify the domain of theoretical constructs relevant to understanding the behaviour change processes necessary to achieve the desired behaviour. These questions were designed to assist implementation researchers and service managers in identifying behaviour change explanations and processes relevant to desired behaviour change. Both open and closed questions were included.

Agreed questions were then piloted in role plays by the theory group and the HSR group. Refined questions were field tested in interviews with healthcare professionals and service managers involved in implementation of EBP. Interviewees were identified and interviewed by members of the theory group. A list of the interviews is given in box 2 .

Feedback on the utility of these interviews in identifying what might be changed in implementing specific guidelines was then considered by members of the theory group and resulted in an amended set of interview questions.

\section{RESULTS}

\section{Identifying theories and theoretical constructs}

128 explanatory constructs drawing on 33 psychological theories were identified (see Appendix 1).

\section{Simplifying into theoretical domains}

Of the 128 constructs, 17 were prioritised as key constructs relevant to interdisciplinary research into understanding and changing behaviour and 14 constructs were prioritised as particularly relevant to changing the behaviour of healthcare professionals. The latter were: reinforcement/reward/ incentives/perceived contingent reward; perceived control/ self-efficacy; intention; action planning/implementation 


\section{Box 2 Pilot interviews}

- Two GPs about implementation of National Service Frameworks for coronary heart disease.

- Two Research and Development leads

- Introduction of an algorithm to assist medical staff in assessing deep vein thrombosis risk.

- Implementation of a care of the dying integrated pathway.

- Two midwives

- National Institute of Clinical Excellence guideline re cardiotocograph monitoring.

- Ward based guideline re ruptured membranes.

- Researcher in Department of Nursing and Midwifery investigating the implementation of an integrated care pathway for community mental health teams.

- GP/implementation researcher re guideline to ensure diabetic retinopathy screening in primary care.

intention; outcome expectancy; goal setting/target setting/ self-monitoring/self-evaluation/feedback; contingencies/ environmental triggers; environmental context/organisational culture; punishment; behavioural control (barriers and facilitators); motivation; attitudes; morale; habit/ routines.

The preliminary list of agreed domains representing the prioritised constructs was:

- Nature of behaviour ("what needs to be changed")

- Knowledge and skills

- Goal intention ("what to aim for")

- Beliefs about consequences

- Beliefs about own capabilities

- Goal plan ("how to achieve change")

- Environment-social

- Environment-physical

- Stress/emotion

- "Other"

Evaluating the importance of the theoretical domains Concerns with the above list included:

- "Social environment" might be better subdivided into organisational and individual influences.

- "Physical environment" was ambiguous in relation to its inclusion of "antecedents" e.g. cues, stimulus conditions, priming.

- "Goal intention" required further clarification.

- "Nature of behaviour" could provide great guidance on the dimensions on which desired behaviours might vary.

- There was no domain dealing with "cognitive processes" e.g. memory.

Checking against the theories and theoretical constructs identified in stage 1, it was agreed that two further construct domains were required: "memory, attention and decision processes" and "beliefs about EBP/guidelines". The latter included professionals' beliefs about guidelines-for example, about their origin, identity and time scale of implementation.
Interdisciplinary evaluation

Discussion of the utility of the domain list for health service researchers led to further refinement. The interdisciplinary group considered that "beliefs about EBP/guidelines" was part of a broader domain of "social/professional role and identity". The "nature of the behaviour" domain was accorded a different order to the rest, as it describes the dependent variable rather than the influencing factors. The constructs assigned to "social/professional role and identity" and to "memory, attention and decision processes" were agreed by the authors after the domains were refined.

\section{Validating the domain list}

Checking that the domain list accurately reflected underlying theoretical constructs through backward validation showed that the theories and theoretical constructs allocated to the domains by the HP group corresponded closely to those generated at stage 1 . This provided reassuring validation of the representativeness of the agreed list of domains. However, some additional constructs were identified for each domain.

The constructs assigned to domains, including those added by the validation exercise, are listed in the second column of table 1. The theoretical domains identified by Fishbein et al are listed (in parentheses) alongside these domains in the first column.

\section{Piloting interview questions}

The questions developed to identify the behaviour change processes likely to be most relevant to implementing specific examples of EBP are shown in the third column of table 1. These were discussed and agreed and subsequently underwent further modification as a result of feedback from in-group role plays and field testing with healthcare professionals and managers. Field interviews provided valuable insights into which domains were most relevant to implementation of particular recommended behaviours and also suggested possible intervention strategies. An illustrative interview is shown in box 3 .

\section{DISCUSSION}

The key objective of the work reported here was to simplify psychological theory relevant to behaviour change and to make it accessible to those involved in EBP implementation. In particular, we have sought to develop a framework, grounded in psychological theory, that would be useful to (and usable by) researchers working with health service managers towards implementation of EBP. The resulting consensus identified 12 theoretical domains that should be considered when seeking explanations of failure to implement EBP and/or designing interventions to achieve improved implementation.

The agreed domains overlap with those identified by Fishbein $e a^{16}$ in the context of promoting HIV preventive behaviour. Since the current consensus was reached independently of the Fishbein et al consensus and in the context of a different set of behaviours, this provides further validation of the domain list generated by the procedures we have described. The first column of table 1 shows the constructs identified by Fishbein et al and how they correspond to our domains. Our list includes four additional domains: "knowledge", "memory, attention and decision processes", "behavioural regulation", and "nature of the behaviour". This might be due to the broader expertise of the UK groups, including the HSR researchers, or due to the particular behaviours we focused on. It may reflect developments in the research literature since the Fishbein et al group met in 1991 while our groups met in 2002/2003. For example, over the last decade there has been considerable new research 
Table 1 Theoretical domains, component constructs, and eliciting questions for investigating the implementation of evidencebased practice

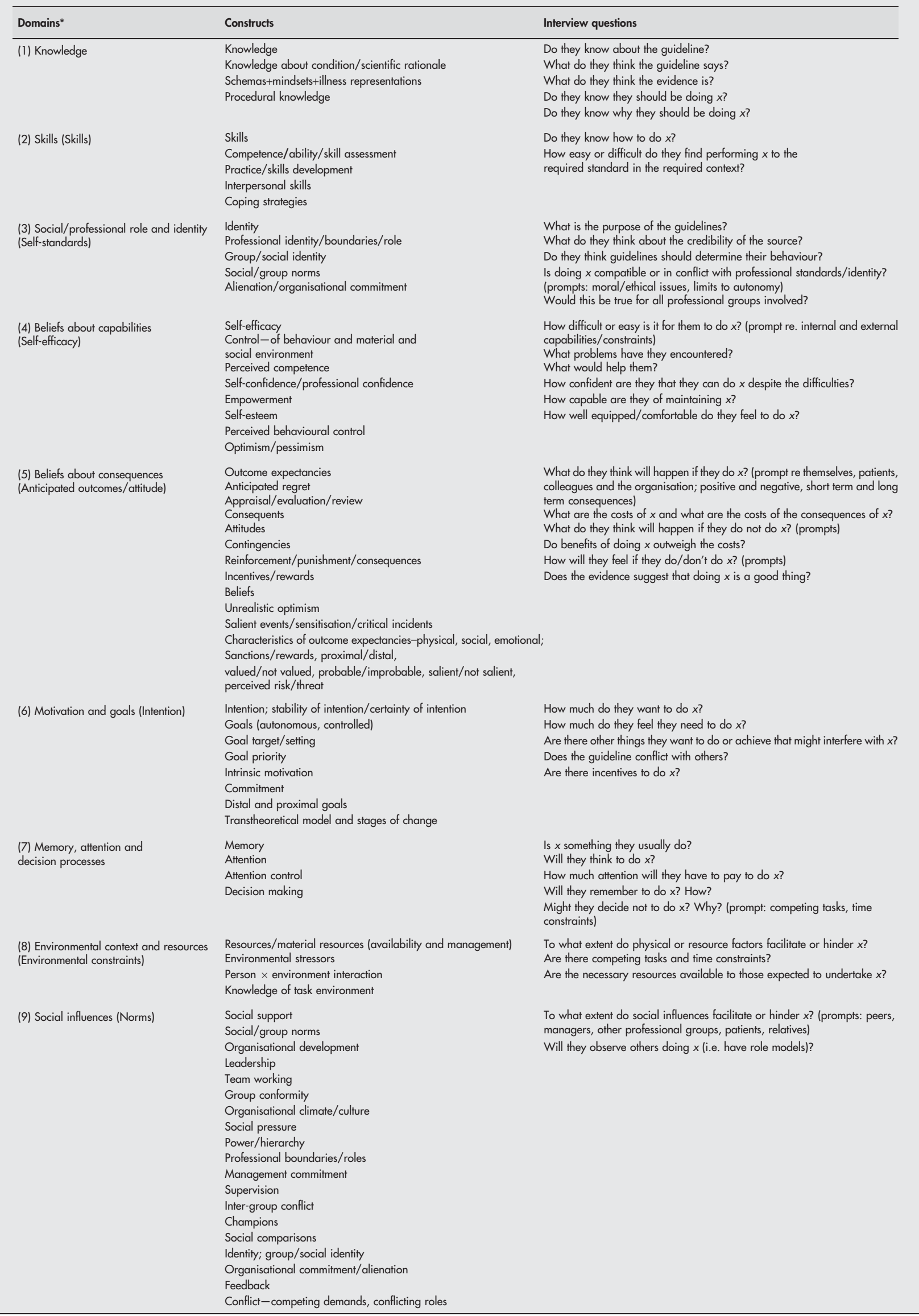




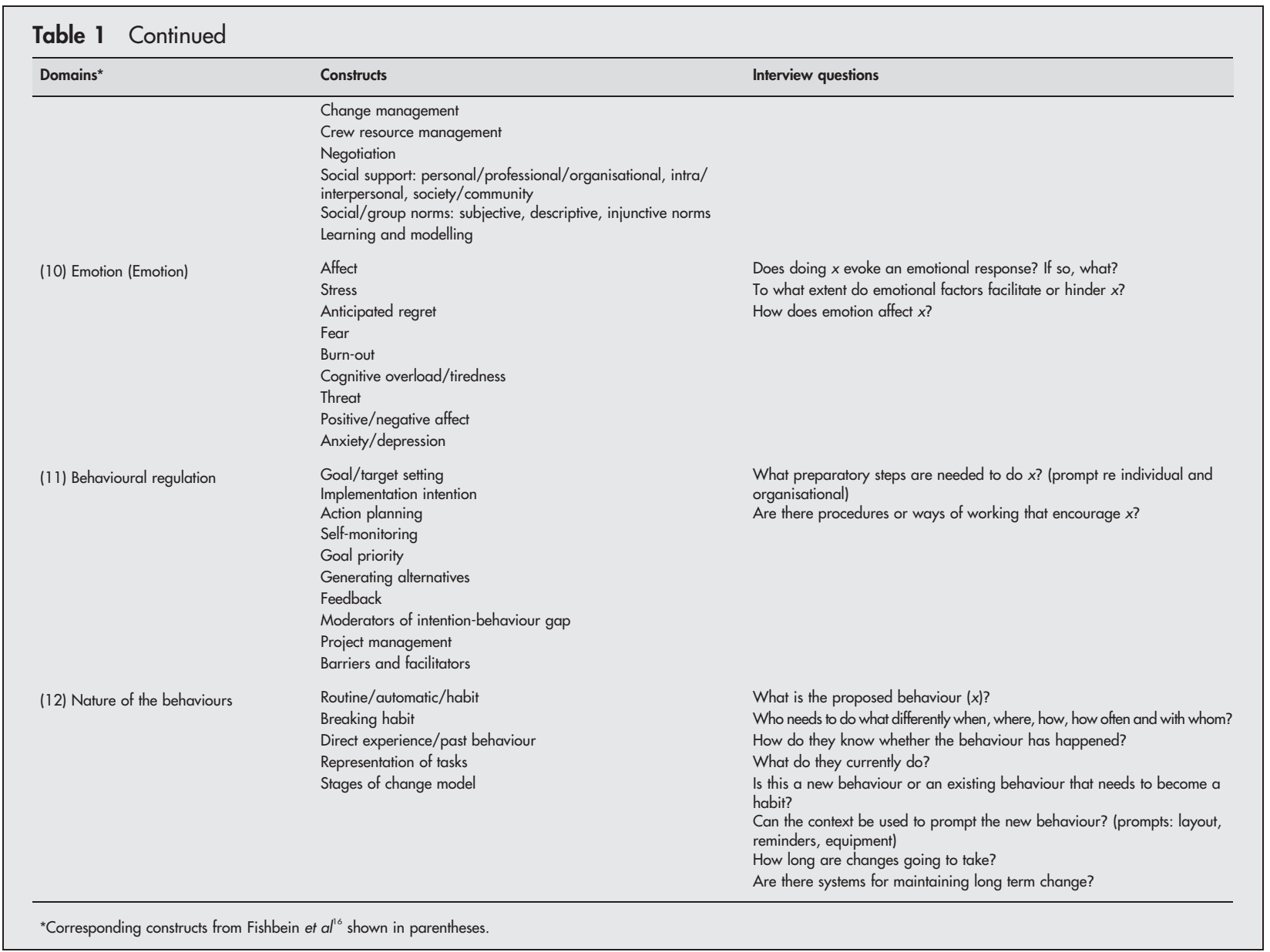

and theory development on planning in behaviour change (for example, Gollwitzer and Brandstätter ${ }^{19}$ ).

However, Fishbein et al suggested that three domains were necessary and sufficient prerequisites for the performance of a specified behaviour-namely, (1) a strong intention to perform the behaviour; (2) no environmental constraints that make it impossible to perform the behaviour; and (3) the skills necessary to perform the behaviour. The remaining five of Fishbein et al's constructs were seen as determinants of intention strength.

Thus, our 12 domains identify key constructs but not the causal processes that link theoretical constructs in a coherent explanation of behavioural regulation or behavioural change. Our domain list provides a guide to relevant explanations of current behaviours and key prompts to behaviour change, rather than an articulated theoretical explanation of a particular set of behaviours.

Both Fishbein et al and our own group emphasised the need to clarify the nature of the behaviour involved. Yet evidence-based guidelines may give poor specificity in defining the necessary behaviours. ${ }^{20}$ Unless there is precision in describing and defining the behaviour that needs to be changed, it will not be possible to identify construct domains that might explain the underlying behavioural processes and the associated opportunities for change.

The interview questions in column 3 of table 1 illustrate the kind of explanation that might be investigated in each domain. Using these questions to explore a behaviour will ensure that no important domain is overlooked, and the results may assist in explaining the behaviour and in designing a behaviour change intervention.
The domains may also provide insights that lead to greater understanding of the processes underlying existing nontheoretically based interventions. For example, educational reminders have been found to help to reduce the frequency of GPs ordering lumbar spine and knee radiographs. ${ }^{21}$ There are several processes that could explain the success of this

\section{Box 3 An illustrative interview}

An interview with a midwife about attempts to reduce routine cardiotocograph (ctg) monitoring during delivery revealed that there were two relevant explanations of the lack of behaviour change. The first was beliefs about consequences. Midwives, particularly those with many years experience, believed that there were substantial risks to mother and baby associated with non-use of ctg monitoring despite evidence indicating otherwise. The second domain that represented a barrier to change was memory, attention and decision processes. The midwife regarded ctg monitoring as an automatic routine activity, cued by the presence of the ctg monitor in the delivery suite. A suggestion was made that, if ctg monitors were removed and accessing one necessitated deliberative processing and effort, the use of ctgs would decrease. This example suggests a two pronged approach to the successful implementation of this evidence: (1) intervene to change beliefs about the consequences of not using the ctg and (2) remove the cue to action provided by the presence of the $\mathrm{ctg}$ in the delivery suite. 


\section{Key messages}

- The implementation of evidence based practice (EBP) depends on human behaviour.

- Interventions to improve the implementation of EBP have had only modest effects.

- Interventions may be improved by drawing on theories of human behaviour.

- Psychology has produced a huge number of such theories, many of which overlap.

- This project aims to simplify and integrate the psychological theories and theoretical constructs of relevance to EBP.

- A consensus approach produced 12 theoretical "domains" as an integrative framework for studying the implementation of EBP.

- The project also produced sets of questions based on this framework that can be used in identifying and understanding problems in EBP.

intervention: the reminders may provide information that enhance knowledge; they may act as cues, creating a facilitative environmental context which triggers behaviour, they may influence attention and memory; they may serve as persuasive communications which lead to changes in beliefs about the consequences of ordering these radiographs; they may function as a social sanction or as contingent feedback, further influencing beliefs in consequences; or they may remind the recipient that other doctors behave differently and may not approve of ordering these radiographs - that is, instigating a process of social influence. Enhancing the understanding of underlying processes will give guidance for developing effective interventions.

While potentially useful, the domain list cannot comprehensively identify all factors that may impede the implementation of EBP. However, barriers that are not obviously psychological such as legal or physical constraints may be mediated by psychological processes represented by our domain list-for example, "beliefs about consequences", "social/professional role and identity", and "environmental context and resources". The use of this explanatory domain list may allow the selection and combination of effective behaviour change techniques and methods, based on over a century of research, to improve implementation of EBP. ${ }^{22} 23$

\section{Authors' affiliations}

S Michie, Department of Psychology, University College London, UK M Johnston, A Walker, Department of Psychology, University of Aberdeen, UK

C Abraham, Department of Psychology, University of Sussex, UK R Lawton, Department of Health Psychology, University of Leeds, UK D Parker, Department of Psychology, University of Manchester, UK

This work was funded by the British Psychological Society with a grant awarded in its 2001 Seminars Research Competition "The scientific understanding of the psychological processes involved in the implementation of evidence based practice (EBP) in health services". The joint meeting of the psychological theory and health services research groups was part funded by the MRC Health Services Research Collaboration.

Members of the "Psychological Theory" Group: Charles Abraham, Debbie Bonetti, Tanya Claridge, Mark Conner, Philippa Davies, Diane Dixon, Derek Johnston, Marie Johnston, Rebecca Lawton, Susan Michie, Dianne Parker, Gwyneth Rees, Noelle Robertson, Paschal Sheeran, Amanda Sowden, Anne Walker, John Weinman.

\section{REFERENCES}

1 Haines A, Donald A. Making better use of research findings. BMJ 1998:317:72-5.

2 Oxman A, Thomson M, Davis D, et al. No "magic bullets": a systematic review of 102 trials of interventions to improve professional practice. Can Med Assoc J 1995; 153:1423-31.

3 Wensing M, Van der Weijden T, Grol R. Implementing guidelines and innovations in general practice: which interventions are effective? $\mathrm{Br} J \mathrm{Gen}$ Pract 1998;48:991-7.

4 Grimshaw J, Thomas R, Maclennan G, et al. Effectiveness and efficiency of guideline dissemination strategies. Health Technol Assess 2004;8:6:1-84

5 Grimshaw JM, Shirran L, Thomas R, et al. Changing provider behavior: an overview of systematic reviews of interventions. Med Care $2001 ; 39: 8: 2: 112-45$

6 Ashford AJ. Behavioural change in professional practice: supporting the development of effective implementation strategies. Report No 88. Centre for Health Services Research, University of Newcastle, 1998

7 Robertson N, Baker R, Hearnshaw H. Changing the clinical behaviour of doctors: a psychological framework. Qual Health Care 1996;5:51-4.

8 Grol R. Beliefs and evidence in changing clinical practice. BMJ 1997;315:418-21.

9 Bandura A. Health promotion from the perspective of social cognitive theory. Psychology and Health 1998;13:623-49.

10 Skinner EA. A guide to constructs of control. J Personality Soc Psychol 1996;71:549-70.

11 Fishbein M, Ajzen I. Belief, attitude, intention and behavior: an introduction to theory and research. Reading, MA: Addison-Wesley, 1975.

12 Bandura A. Self-efficacy: towards a unifying theory of behavioural change. Psychol Rev 1977;84:191-215.

13 Triandis HC. Interpersonal behaviour. Monterey, CA: Brooks-Cole, 1977.

14 Kanfer R, Kanfer FH. Goals and self-regulation: applications of theory to work settings. In: Maehr ML, Pintrick PR, eds. Advances in motivation and achievement. Greenwich, CT: JAI Press, 1991;7:287-326.

15 Becker MH. The health belief model and personal health behavior. Health Educ Monogr 1974;2:324-508.

16 Fishbein M, Triandis HC, Kanfer FH, et al. Factors influencing behaviour and behaviour change. In: Baum A, Revenson TA, Singer JE, eds. Handbook of health psychology. Mahwah, NJ: Lawrence Erlbaum Associates, 2001:3-17

17 Ajzen I. The theory of planned behavior. Organis Behav Human Decis Proc 1991;50:179-211.

18 Walker A, Grimshaw JM, Johnston M, et al. "PRIME-PRocess modelling in ImpleMEntation research: selecting a theoretical basis for interventions to change practice". BMC Health Serv Res 2003:3-22.

19 Gollwitzer PM, Brandstätter V. Implementation intentions and effective goal pursuit. J Personality Soc Psychol 1997;73:186-99.

20 Michie S, Johnston M. Changing clinical behaviour by making guidelines specific. BMJ 2004;328:343-5

21 Eccles M, Grimshaw J, Walker A, et al. Changing the behaviour of healthcare professionals: the use of theory in promoting the uptake of research findings. $J$ Clin Epidemiol 2004 (in press).

22 Hardeman W, Griffin S, Johnson M, et al. Interventions to prevent weight gain: a systematic review of psychological models and behaviour change methods. Int J Obesity 2000;24:131-43.

23 Kanfer FH, Goldstein AP. Helping people change: a textbook of methods. 4th ed. London: Allyn and Bacon, 1991.

24 Myers D. Social psychology. 7th ed. New York: McGraw Hill, 2002

25 Al-Damouk M, Pudney E, Bleetman A. Hand hygiene and aseptic technique in the emergency department. J Hosp Infect 2004;56:137-41.

26 Chief Medical Officer's Report. Getting ahead of the curve: a strategy for combating infectious diseases. London: CMO, 2002.

\section{APPENDIX 1 PSYCHOLOGICAL THEORIES IDENTIFIED IN STAGE 1}

\section{MOTIVATION THEORIES}

- Theory of planned behaviour (+ theory of reasoned action, protection motivation theory, health belief model)

- Social cognitive theory

- Locus of control theories

- Social learning theory

- Social comparison theory

- Cognitive adaptation theory

- Social identity theory

- Elaboration likelihood model

- Goal theories

- Intrinsic motivation theories 
- Self-determination theory

ORGANISATION THEORIES

- Attribution theory

- Effort-reward imbalance

- Decision making theories (e.g. social judgment theory, "fast and frugal" model, systematic versus heuristic decision making)

- Fear arousal theory

More information about these theories is available from Myers. $^{24}$

\section{ACTION THEORIES}

- Learning theory

- Operant theory

- Modelling

- Self-regulation theory

- Implementation theory/automotive model

- Goal theory

- Volitional control theory

- Social cognitive theory

- Cognitive behaviour therapy

- Transtheoretical model

- Social identity theory

- Demand-control model

- Diffusion theory

- Group theory (e.g. group minority theory)

- Decision making theory

- Goal theory

- Social influence

- Person situation contingency models

\section{APPENDIX 2 GLOSSARY OF TERMS}

Framework: a structure composed of parts framed together. Theory: a system of ideas or statements held as an explanation or account of a group of facts or phenomena.

Theoretical construct: a concept specially devised to be part of a theory.

Domain: an area of interest; a sphere of thought, action or knowledge.

Theoretical domain: a group of related theoretical constructs. Cue: a stimulus or signal to perception, articulation, or other physiological response.

Stimulus: an event (whether internal or external to the organism) which gives rise to a reaction.

\section{Register now!}

10th European Forum on Quality Improvement in Health Care

13-15 April 2005, ExCel Conference Centre, London

For further information on how to register please go to:

http://www.quality.bmipg.com 